\title{
Kernos
}

Revue internationale et pluridisciplinaire de religion grecque antique

8 | 1995

Varia

\section{L'argumentation d'Hérodote concernant les emprunts faits par les Grecs à la religion égyptienne}

\section{Gerasimoula Zographou}

\section{(2) OpenEdition}

\section{Journals}

\section{Édition électronique}

URL : http://journals.openedition.org/kernos/602

DOI : $10.4000 /$ kernos.602

ISSN : 2034-7871

\section{Éditeur}

Centre international d'étude de la religion grecque antique

\section{Édition imprimée}

Date de publication : 1 janvier 1995

Pagination : 187-203

ISSN : 0776-3824

\section{Référence électronique}

Gerasimoula Zographou, «L'argumentation d'Hérodote concernant les emprunts faits par les Grecs à la religion égyptienne », Kernos [En ligne], 8 | 1995, mis en ligne le 11 avril 2011, consulté le 30 avril 2019. URL : http://journals.openedition.org/kernos/602 ; DOI : 10.4000/kernos.602 


\section{L'argumentation d'Hérodote concernant les emprunts faits par les Grecs à la religion égyptienne}

Les influences culturelles ont toujours constitué un facteur d'évolution aussi inévitable que fécond. La civilisation grecque n'a pas échappé à cette règle. En tant que commerçants et marins actifs, les Grecs ont très tôt créé des relations avec leurs voisins, notamment avec les Phéniciens et les Égyptiens; avec ces derniers, les contacts datent au moins de l'époque mycénienne ${ }^{1}$.

Dans le deuxième livre des Histoires, Hérodote, comme on le sait, disserte longuement sur la civilisation égyptienne et les influences qu'elle aurait exercées sur la civilisation grecque. Selon lui, ces influences concernent plusieurs domaines de la vie grecque, notamment l'activité scientifique et le domaine de la religion. C'est sur ce dernier que je porterai mon attention.

En effet, ce qui m'intéresse plus particulièrement c'est de voir si, et dans quelle mesure, l'historien a raison lorsque il parle d'emprunts faits par les Grecs à la religion égyptienne. Je dois d'emblée souligner que le thème fait partie d'une étude philologique plus étendue qui se réfère à la méthode d'argumentation d'Hérodote. C'est pourquoi il sera examiné d'un point de vue purement

* Je tiens à remercier ici Mme S. Constantinidis et MM. L. Couloubaritsis, A. Motte et P. Noussis pour leur aide.

1 Sur les contacts des Grecs avec les régions de l'Est de la Méditerranée durant l'époque mycénienne, voir A. SEVERYNS, Grèce et Proche-Orient avant Homère, Bruxelles, 1960, p. 121-134; J.B. BURY - R. MEIGGS, A History of Greece to the Death of Alexander the Great, LondonBasingstoke, $1975^{4}$, p. 64 ; on trouvera également dans ce dernier ouvrage un aperçu exhaustif des relations gréco-phéniciennes après la chute de Mycènes. D’autre part, des découvertes archéologiques attestent que les relations avec l'Égypte remontent à la civilisation créto-minoenne: A. EVANS, The Palace of Minos, New York, 1964, vol. I, p. 192-208, vol. II, p. 448-450, 453, 474, vol. III, p. 104, 112, 168; J.S. PENDLEBURY, The Archaeology of Crete, An introduction, London, 1967 [1939]; J. VERCOUTTER, Essat sur les relatlons entre Égyptiens et Prébellènes, Paris, 1954; F. SCHACHERMEYR, Agdis und Orient: Die iberseetschen Kulturbeztebungen von Kreta und Mycene mit Ägypten, der Levante und Kleinasien unter bes. Berilcksichtigung des $2 \mathrm{Jt} . v$. Cbr., Wien, 1967; aux p. 44-49, l'auteur examine les influences réciproques entre civilisations minoenne et égyptienne. On se reportera aussi à l'importante contribution d'A.B. LLOYD, Herodotus Book II, Introduction, Leiden, 1975, p. 1-60, qui étudie la présence des Grecs en Égypte, depuis l'époque de l'Âge de Bronze jusqu'à l'époque d'Hérodote. Selon B.C. DIETRICH, The Origins of Greek Religion, Berlin-New York, 1974, p. 5-6, il existe des contacts culturels entre la Grèce et la Proche-Orient depuis le Néolithique. En ce qui concerne les relations du monde mycénien avec l'Égypte, voir H. BENGTSON, Griechiscbe Gescbicbte von den Anfangen bis in die römische Kaiserzeit, München, $1977^{5}$, p. 48; J.T. HOOKER, Mycenaean Greece, London, 1976, p. 50-54, 67-69, 115. 
littéraire : il s'agit de discerner comment l'auteur des Histoires argumente et comment il prouve son point de vue.

L'intérêt d'Hérodote pour la civilisation égyptienne est placée d'emblée

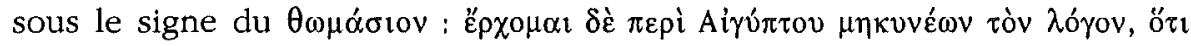

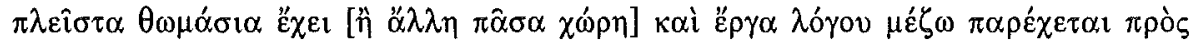

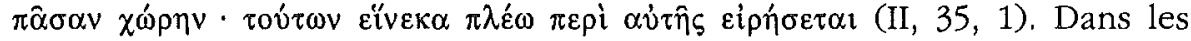

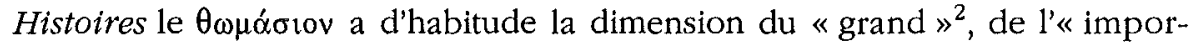
$\operatorname{tant} »^{3}$, du «merveilleux $»^{4}$. Même les différences ou les ressemblances entre la civilisation égyptienne et les autres civilisations éveillent la curiosité de l'historien et lui fournissent des éléments de comparaison pour démontrer la supériorité de la civilisation égyptienne.

Cette argumentation apparaît surtout aux chapitres 35-98, où l'historien décrit les coutumes et les mœurs, essentiellement celles de l'Égypte contempo-

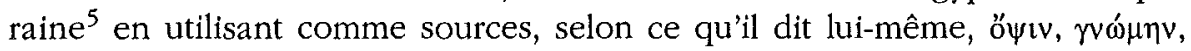

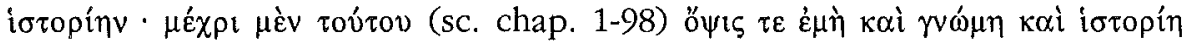

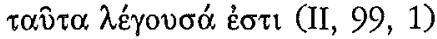

Hérodote affronte les relations entre civilisation égyptienne et grecque à travers une triple logique : celle de l'opposition, de l'analogie et de leur combinaison (analogie plus opposition) ${ }^{6}$.

Commençant par la constatation selon laquelle les Égyptiens $\tau \grave{\alpha} \pi 0 \lambda \lambda \dot{\alpha}$

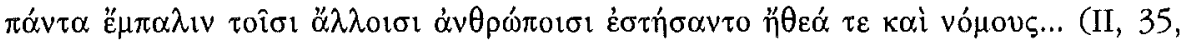
2), il consacre les premiers chapitres (35.36) aux coutumes qui caractérisent, d'une part, les Égyptiens, par exemple, les rapports entre hommes et femmes et, d'autre part, les autres peuples.

Ainsi apprenons-nous, par exemple, qu'en Égypte, les femmes vont au marché et font du commerce, tandis que les hommes restent à la maison et tissent; les hommes soulèvent les charges différemment des femmes; les femmes ne peuvent devenir prêtresses d'aucun dieu, ni masculin ni féminin, au contraire des hommes; le fils n'a aucune obligation de s'occuper de ses parents, s'il ne le veut pas, tandis que la fille est tenue de le faire, même si elle ne le veut pas.

Si nous mentionnons ces principales particularités dans les relations hommes-femmes qui ont attiré l'attention d'Hérodote c'est parce qu'il s'agit

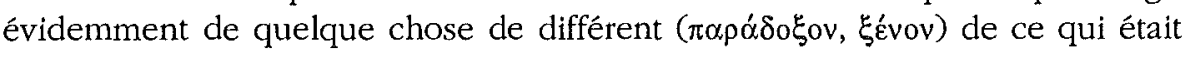

2 Voir par exemple : II, 6 sq.; II, 124-125, 148-149.

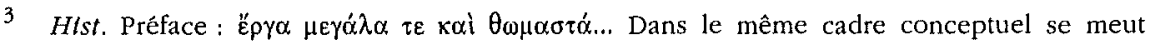
aussi, je pense, sa longue référence à certains rois égyptiens, comme Sésostris (II, 102 sq.)

4 Voir par exemple II, 17 sq., où il s'occupe du phénomène étrange de l'inondation du Nil.

5 Voir A.B. LLOYD, Introduction, op. cit. (n. 1), p. 84. Selon J. McEvoY, Platon et la sagesse de l'Égypte, in Kernos, 6 (1993), p. 257, n. 23, dans le livre II, Hérodote décrit l'Égypte de l'époque saitique (environ 663-525 av. J.-C.).

6 En ce qui concerne l'analogie et l'opposition comme formes de l'argumentation caractérisant la pensée grecque ancienne, voir G.E.R. LLOYD, Polarity and Analogy, Two types of Angumentation in Early Greek Thougbt, Bristol, 1987 [Cambridge, 1966]. 
pratiqué en Grèce. En effet, on sait qu'à Athènes pendant l'antiquité les hommes fréquentaient le marché, tandis que les femmes restaient à la maison ${ }^{7}$, la quenouille était l'affaire des femmes contrairement à ce qui se passait en Égypte $^{8}$. L'assistance aux parents était une obligation pour les fils et non pour les filles ${ }^{9}$. En ce qui concerne le thème du sacerdoce, qui nous intéresse particulièrement, A. Lloyd observe : " no woman in Egypt performs the divine cult of any diety or occupied the pre-eminent rôle in worship which would make her equivalent to what he would call a ieprín in Greece ${ }^{10}$.

Hérodote constate les différences entre la civilisation égyptienne et la civilisation des autres peuples, en particulier la civilisation grecque, tantôt en apportant des preuves ${ }^{11}$ de ce qu'il avance, tantôt, comme c'est le cas généralement, sans preuves. Rappelons quelques exemples significatifs : les prêtres égyptiens se rasent tandis que les prêtres des autres peuples (et des Grecs) ne le font pas nécessairement ${ }^{12}$; les Égyptiens se nourrissent principalement de viande, les Grecs, de céréales ${ }^{13}$; le pain est pétri avec les pieds et l'argile avec les mains contrairement aux habitudes grecques ${ }^{14}$. Ils écrivent de droite à gauche, alors que les Grecs font l'inverse ${ }^{15}$. Dans la suite (II, 37 sq.), Hérodote décrit les règles religieuses et les cérémonies des Égyptiens. Ici, on ne trouve pas l'opposition radicale que nous avons constatée dans les chapitres précédents, où abonde l'utilisation de conjonctions d'opposition $\mu \varepsilon ́ v . . . \delta \varepsilon$. Cependant, il est manifeste qu'il sous-entend de nouveau une comparaison antithétique, qui favorise la civilisation égyptienne. Cela est indiqué tant par la phrase inaugurale

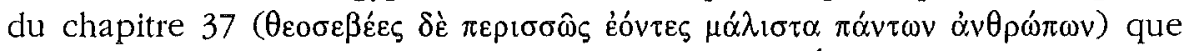
par d'autres expressions qui soulignent le respect des Égyptiens pour les règles de la vie religieuse (manifestement en opposition avec celles utilisées par

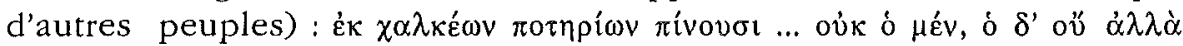

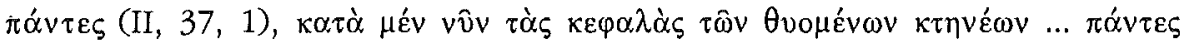

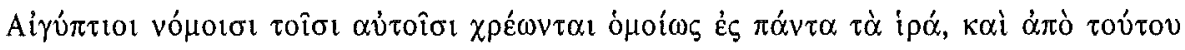

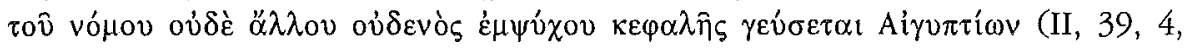
entre autres).

7 R. FLACELIÈRE, La vie quotidienne en Grèce au siècle de Périclès, Paris, 1959, p. 87 sq.; W.K. LACEY, The Family in Classical Greece, London-Southampton, 1968, p. 167-168.

8 A.B. LLOYD, Herodotus Book II, Commentary 1-98, Leiden, 1976, p. 148.

9 FLACELIÈRE, op. cit. (n. 7), p. 103; LACEY, op. cit. (n. 7), p. 116 sq.

10 Commentary 1-98, op. cit. (n. 8), p. 151.

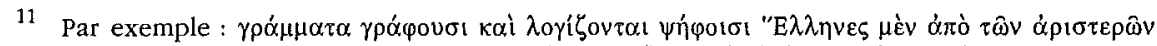

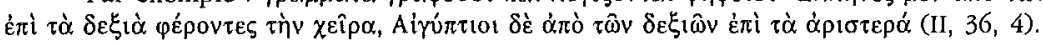

12 A.B. LLOYD, Introduction, op. ctt. (n. 1), p. 142 : « in Greece long hair was not infrequent for priestly officials $»$.

13 H. BLÜMNER, Technologie und Terminologie der Gewerbe und Küste bei Griechen und Römern, vol. I., Hildesheim, 1969 ([1912²], p. 51 sq.; FLACELIÈRE, op. cit. (n. 7), p. 207.

14 BLÜMNER, op. cit. (n. 13), p. 65 sq.

15 Voir II, 36, 4. 
Qu'Hérodote fasse ces observations en ayant en vue également les différentes habitudes religieuses des Grecs, nous pouvons le constater en étudiant attentivement le texte. Par exemple, les prêtres égyptiens rasent tout leur corps (II, 37, 2), tandis que les prêtres grecs ont souvent des cheveux longs ${ }^{16}$; en Égypte, chaque dieu compte beaucoup de prêtres (II, 37, 5), à l'inverse de ce qui se passe en Grèce ${ }^{17}$; la tête de l'animal qu'on sacrifie est considérée comme impure : on la charge de malédictions et on la jette dans le fleuve et ce, uniquement lorsqu'il n' y a pas de marchands grecs passant par là pour l'acheter (II, 39, 2-3), fait qui signifie que les Grecs ne connaissent pas de telles superstitions; les entrailles de l'animal sacrifié sont laissées par les Égyptiens dans son corps (II, 40,2), tandis que les Grecs les enlèvent et les mangent ${ }^{18}$; en Égypte les vaches sont considérées comme des animaux sacrés, ce qui n'est pas le cas en Grèce (II, 41, 2-3), etc.

D'autres exemples donnent un aperçu des nombreuses différences entre les deux peuples : en Égypte, on considère le porc comme un animal impur qu'on évite de sacrifier (II, 47, 1-2); inversement, en Grèce, c'est un animal des plus courants qu'on offre en sacrifice, en l'honneur de la déesse Déméter, principalement lors des mystères éleusiniens, où il sert à la purification ${ }^{19}$. Dans la religion égyptienne, il n'y a pas de héros (II, 50, 3) contrairement à ce qui se passe dans la religion grecque. En Égypte, on n'admet pas que la divination soit une propriété de l'homme, mais seulement de quelques dieux (II, 83); en Grèce, comme on sait, les devins les plus connus ne sont pas des dieux : Calchas, Tirésias, Cassandre, la Pythie ${ }^{20}$. Les dieux égyptiens ne prennent pas non plus de formes humaines et ne se mêlent pas aux mortels (II, 142,3) et II, $143,4)$, comme en Grèce. Bien plus, les Égyptiens ne croient pas qu'un homme puisse être né d'un dieu (II, 143, 4); au contraire, les Grecs considèrent tout à fait naturel l'accouplement des dieux avec les mortelles (ou l'inverse) qui donne naissance à des enfants mortels ou à des demi-dieux.

16 W. BURKERT, Greek Religion, Archaic and Classical, trad. par J. Raffan, Oxford, 1985 [l'édition originale allemande date de 1977], p. 97.

17 Cf. R. GARLAND, Priests and Power in Classical Athens, in M. BEARD - J. NORTH (eds), Pagan Priests. Religion and Power in the Ancient World, London, Duckworth, 1990, p. 77. Sur ce thème et sur les iepeî̧ en général, on peut voir aussi BURKERT, op. cit. (n. 16), p. 95-8.

18 Il., I, 464; Od., III, 9. Voir aussi F. HARTOG, Le bœutf " autocuiseur" " et les boissons d'Arès, in M. DETIENNE - J.-P. VERNANT, La cuisine du sacrifice en pays grec, Paris, 1979, p. 261-264; J. GOULD, On making sense of Greek Religion, in P.E. EASTERLING - J.V. MUIR (eds), Greek Religion and Society, Cambridge, 1985, p. 17.

19 P. STENGEL, Die griechischen Kultusaltertumer, München, 1920, p. 121 sq.; M.P. NILSSON, Gescbicbte der griechischen Religion ( $G G r R$ ), vol. I., München, 1955², p. 213, 321; G. MYLONAS, Eleusis and the Eleusinian Mysteries, Princeton, New Jersey, 1961, p. 201, 203, 205, 249-250; W. BURKERT, Homo Necans. Interpretationen altgriechischer Opferriten und Mytben, Berlin-New York, 1972, p. 303; H.W. PARKE, Festivals of the Athentans, London, 1977, p. 62 sq.; M. DETIENNE, Pratiques culinaires et esprit de sacrifice, in La cuisine du sacrifice, op. cit. (n. 18), p. 17 et, Vlolentes " eugénies ». En pleines Thesmophortes: des femmes couvertes de sang, ibid., p. 192-193.

20 Voir GARLAND, art. cit. (n. 17), p. 82 sq. 
Ces constatations autorisent de premières conclusions. Sur base d'une logique des couples opposés qui régit la pensée archaïque, tels que nous les

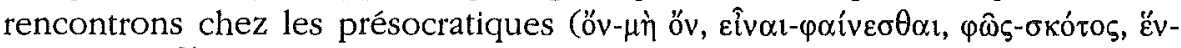
$\pi \mathrm{o} \lambda \lambda \alpha_{\alpha}$, etc.) ${ }^{21}$ et suivant une démarche qui va du particulier (hommes-femmes égyptiennes) au général (Égyptiens-autres peuples), Hérodote condense, dans les deux premiers chapitres $(35,36)$, les spécificités de la civilisation égyptienne par rapport à d'autres civilisations ${ }^{22}$. L'historien réussit de cette façon à nous en donner une image générale et prépare ses lecteurs à de plus amples informations en matière de religion, domaine qui l'intéresse tout particulièrement. Déjà se manifeste son intention de montrer, à travers les oppositions, que la civilisation égyptienne est, en fin de compte, supérieure aux autres et que les autres peuples lui doivent beaucoup. ${ }^{23}$ Lorsqu'Hérodote décèle en effet des analogies et des ressemblances entre les deux civilisations, il les interprète bien comme des emprunts dans un sens seulement, allant de l'Égypte à la Grèce, ce qui manifeste la supériorité de la civilisation égyptienne.

C'est ainsi que dans le cadre religieux, les Égyptiens se représentent Pan comme les Grecs (II, 46, 2), mais il n'est pas question d'influence grecque; ils organisent la fête en l'honneur de Dionysos presque de la même façon que les Grecs (II, 48, 2); néanmoins, Hérodote défend une influence égyptienne. Les pratiques de divination à Thèbes en Égypte et à Dodone sont semblables, car elles ont une origine commune, naturellement égyptienne, comme d'ailleurs la divination par l'examen des victimes (II, 57, 3). Ce sont les Égyptiens qui, les premiers, célèbrent de grandes fêtes religieuses nationales, des processions faisant cortège aux dieux et accompagnant des offrandes; les Grecs, toujours selon Hérodote, ont appris d'eux la coutume (II, 58); pourtant, des indices montrent que les Grecs avaient déjà ces coutumes depuis l'âge de Bronze et que leur lieu de provenance était la Crète ${ }^{24}$. Presque tous les hommes, à l'exception des Égyptiens et des Grecs, s'unissent aux femmes dans les lieux saints et entrent dans un sanctuaire sans s'être lavés au préalable (II, 64, 1); de

21 G. LLOYD, op, ctt. (n. 6), p. 15 sq.

22 A.B. LLOYD, Introduction, op. ctt. (n. 1), p. 152, parle d'une sur-schématisation dans laquelle se réfugie ici l'historien pour établir des couples d'oppositions semblables à ceux des Présocratiques. Par exemple, il n'est pas absolument certain que seules les femmes fréquentaient le marché, tandis qu'il n'y a pas d'indices montrant que les femmes portaient les charges différemment des hommes : A.B. LLOYD, Commentary 1-98, op, ctt. (n. 8), p. 149; W.W. HOW - J. WELLS, $A$ Commentary on Herodotos, vol. I, (Books I-IV), Oxford, 1964 [1912], p. 180.

23 Il convient de se rapporter à l'analyse détallée de C. FROIDEFOND, Le mirage égyptien dans la littérature grecque d'Homère à Aristote, Aix-en-Provence, 1971, p. 129-136, notamment pour les emprunts que fait Hérodote à la tradition concernant les catalogues des $\theta \omega \mu \alpha \sigma o t \alpha$, des vó $\mu \iota \mu \alpha$, ainsi que pour sa propre contribution à la création de «tableaux contrastés » dont il se sert au livre II.

24 Voir A.B. LlOYD, Commentary 1-98, op. cit. (n. 8), p. 266-267, où l'on peut trouver une bibliographie sur le thème. Selon B. DIETRICH, Tradition in Greek Religion, Berlin-New York, 1986 passim, des traditions dès l'époque néolithique ont survécu dans la religion grecque postérieure. Comme il dit, "the Neolithique, content of certain festivals of Artemis and Demeter, like the universal Thesmophoria has already been noted ", p. 69. Voir aussi BURKERT, Greek Religion, op. cit. (n. 16), p. 13. 
nouveau, c'est une influence égyptienne qui est suggérée puisque, dit Hérodote, ce sont les Égyptiens qui, les premiers, se sont fait une loi de ne pas s'unir à des femmes dans les sanctuaires.

Partant de ces données on peut se poser la question de savoir comment avec autant de différences, souvent essentielles, comme par exemple les relations entre les dieux et les hommes, il est possible de parler à tel point d'emprunts. Et à supposer qu'on puisse établir des emprunts, pourquoi ceux-ci proviendraient-ils toujours des Égyptiens, alors que les découvertes archéologiques prouvent des relations réciproques depuis l'époque minoenne ${ }^{25}$ ? Que de telles questions soient fondées, cela ressort de ce qu'on peut constater à propos des cas intermédiaires reconnus par Hérodote où il reconnaît la similitude des deux civilisations. Par exemple, au chapitre 42 , nous lisons que les Égyptiens représentent Isis sous forme féminine avec les cornes de vache, comme les Grecs.

À première vue, l'analogie qui est ici signalée, laisse entendre une influence grecque. Pourtant, Hérodote refuse à ceux-ci la capacité de respecter les vaches ${ }^{26}$. Le cas d'Héraclès mérite un intérêt particulier. Selon Hérodote, les Égyptiens aussi bien que les Grecs possèdent leur Héraclès (analogie) alors qu'il s'agit en fait de deux personnages différents (opposition). L'Héraclès des Égyptiens est un des douze dieux, tandis que celui des Grecs est simplement le rejeton d'Amphitryon (II, 43, 1-2). Malgré cela, l'historien exprime une certitude absolue concernant un emprunt de la religion grecque à la religion égyptienne :

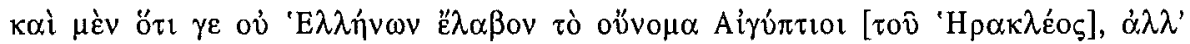

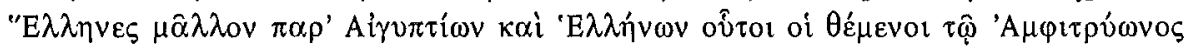

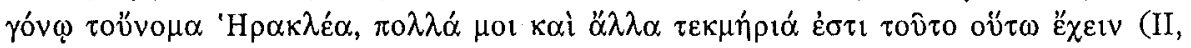
$43,2)$. Il est significatif qu'il formule ici tout d'abord sa position et essaie ensuite de la prouver.

Voyons à quel degré le point de vue d'Hérodote est justifié. Commençons par le mot oúvora. Le sens exact de ce terme a provoqué de nombreuses discussions : signifie-t-il simplement övo $\mu \alpha(=\mathrm{nom})^{27}$ ou s'agit-il d'un terme plus complexe, où l'historien veut dire que « the Greek deities were defined, and their attributes and cult settled, by Egypt ${ }^{28}$ ? Je n'ai pas l'intention de

25 Cf. supra, n. 1.

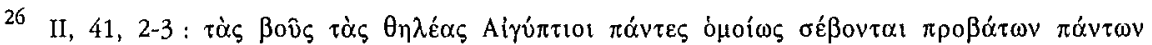

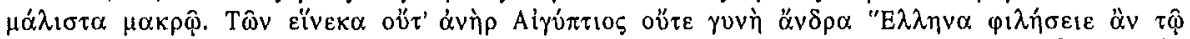

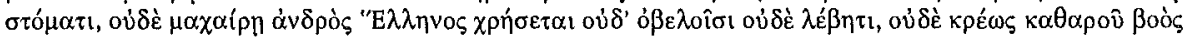

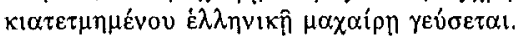

27 Voir A.B. LlOYD, Commentary 1-98, op. cit. (n. 8), p. 203-204, où l'on peut trouver aussi la bibliographie sur le thème; J.A.S. EVANS, Herodotus, Boston, 1982, p. 41; McEVOY, art. ctt. (n. 5), p. 254.

28 How-WELLS, op, ctt. (n. 22), p. 191. Semblables opinions, selon lesquelles le mot ou้vo $\mu \alpha$ signifie "personnalité ", ont été exprimées par d'autres chercheurs, comme I.M. LINFORTH, Greek Gods and Foretgn Gods in Herodotus, in UCPCPh, 9 (1926), p. 1-25, particulièrement p. 18 sq. et $C P h$, 35 (1940), p. 300-301; K. VON FRITZ, Die Grlechiscbe Gescbichtschreibung, vol. I : Von den Anfangen bis Thukydides (Anmerkungen), Berlin, 1967, p. 99; H.W. PARKE, The Oracles of Zeus, 
rallumer le débat. Je me limiterai seulement à quelques indications : tout d'abord, dans le texte des Histoires, on le rencontre en général dans sa signification originale et principale, c'est-à-dire au sens de nom propre d'une personne, d'une nation etc. ${ }^{29}$; ensuite, Hérodote utilise, dans d'autres cas, également des critères linguistiques pour démontrer des parentés et des influences ${ }^{30}$; enfin, le mot oüvo $\mu \alpha$ ne se rapporte pas seulement à Héraclèsdieu mais aussi à Héraclès-mortel. Dans ce dernier cas la deuxième interprétation requiert quelques nuances ${ }^{31}$. Or, s'il en est ainsi, la thèse de l'historien selon laquelle le nom d'Héraclès est d'origine égyptienne ne semble pas

Oxford, 1967, p. 57. FROIDEFOND, op. cit. (n. 23), p. 151 sq. donne au mot un sens plus abstrait et large : les Grecs, dit-il, ont emprunté à l'Égypte « l'idée d'une manifestation multiple et polymorphe de la puissance divine, d'une différenciation significative de cette puissance dans ses rapports avec les hommes, bref, l'essentiel de leur polythéisme ». D'autre part, dans son article Herodote aber die Namen der Gotter: Polytheismus als bistortscbes Problem, in $M H, 42$ (1985), p. 121-132 et surtout, p. 130, W. BURKERT soutient que, chez Hérodote, le mot oúvou $\alpha$ ne signifie pas la dénomination comme telle, mais la capacité que les Grecs ont acquise dans leurs rapports avec les Égyptiens de différencier les dieux entre eux en opposition avec l'ancienne unité indivisible du divin qu'utilisaient les Pélasges. Comme il le dit: « es geht nicht um einzelne, punctuelle Entsprechung von Lautgebilden, sondern darum, dass ein System von Bedeutungen ein anderes eindeutig abbildet ". Par ailleurs, comme l'explique J. RUDHARDT dans son étude De l'attitude des Grecs à l'égard des religions étrangères, in RHR, 209 (1992), p. 228, "donner un nom au dieu, ce n'est pas le rencontrer pour la première fois; c'est alors qu'on l'a déjà rencontré, lui reconnaître assez de traits caractéristiques pour l'identifier ". Cependant, même dans le cas où l'on s'accorderait à de telles positions, on peut formuler des réserves quant à la certitude des jugements d'Hérodote même si les spécialistes des religions ne sont pas d'accord sur la création et l'évolution de l'antique religion grecque à laquelle, selon les points de vue présentés ci-dessous, est lié le mot oüvo $\mu \alpha$ : voir par exemple, M.P. NILSSON, The Minoan-Mycenaean Religion and its Sturvivals in Greek Religion, New York, $1971\left[1950^{2}\right]$, p. 1-30, qui pense que la religion grecque est un mélange de la religion grecque originale (c'est-à-dire de la religion des populations préhélléniques autochtones et des Grecs-Indo-européens-Mycéniens) et de la religion minoenne; DIETRICH, Origins, op. cit. (n. 1), qui cherche à démontrer que la religion grecque a été créée sous l'influence des religions de l'Est; BURKERT, Greek Religion, op. cit. (n. 16), p. 19, qui parle d'une synthèse entre une sous-couche autochtone (préhéllénique) et une influence indo-européenne, tout en se refusant à déterminer ce qui est d'origine grecque et ce qui provient d'influences orientales; R. MUTH, Einfithrung in die griechische und römische Religion, Darmstadt, 1988, p. 28, qui accepte la thèse de base de BURKERT sans les réserves qu'il avance. D'autre part, Hérodote pose le problème du panthéon : de quelle époque parle-t -il ? Le panthéon de la religion minoenne diffère de celui de la religion mycénienne, lui-même différant du panthéon de l'époque classique. On peut se référer à titre indicatif à HOOKER, op. cit. (n. 1), p. 205 sq.; BURKERT, Greek Religion, op. cit. (n. 16), p. 39-

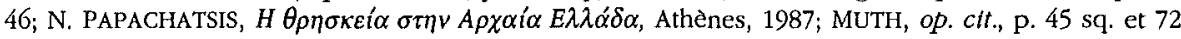
sq. Enfin, peut-on se contenter du rapport entre le nom et les traits spécifiques et négliger une autre variante importante, comme celle du lieu? Pour une interrogation programmatique, voir J.-P. VERNANT, Mythe et société en Grèce ancienne, Paris, Maspero, 1974.

29 Voir J.E. POWELL, A Lexicon to Herodotus, $1960^{2}$ [Cambridge 1938], p. 278, (s.v. övo $\left.\mu \alpha\right)$; cf. H. LIDDELL - R. SCOTT, A Greek-English Lexikon (Revised by Sir H. JONES), Oxford, $1966^{9}$, p. 1232 (s.v. óvo $\alpha$ ); c'est la signification que POWELL attribue au mot dans ce passage concret.

30 II, 42, 4-5 : les Ammoniens sont parents avec les Égyptiens parce qu'ils doivent leur nom au dieu égyptien Zeus Ammon; II, 105: les Colchidiens sont d'origine égyptienne parce que, entre autres, leur langue ressemble à cette des Égyptiens.

31 On peut voir P.E. LEGRAND (éd. et trad.), Hérodote, Histoires Livre II, Euterpe, Paris 1963 [1930], p. 96, n. 3, et p. 103, n. 3, qui, dans des contextes analogues, donne au mot oủvor $\alpha$ des sens différents, parmi lesquels figure celui de «nom ». 
valable, car il est plus probable que le nom soit grec. Il provient de " $\mathrm{H} \rho \alpha+$ $\kappa \lambda \varepsilon ́ o \varsigma$, que Farnell interprète comme «the glory of Hera ${ }^{32}$.

D'autre part, toujours selon Hérodote, Héraclès grec était fils d'Amphitryon et d'Alcmène qui étaient par leurs ancêtres d'origine égyptienne, car tous deux étaient descendants de Persée, lui-même descendant d'Aigyptos et de Danaos $^{33}$. C'est pour deux raisons, je suppose, que l'historien préfère cette version à celle plus courante, qui fait d'Héraclès le fils de Zeus ${ }^{34}$ : d'abord, parce qu'il pense que de cette façon il prouve l'origine égyptienne du mythe, en évitant tout élément qui pourrait le conduire dans une autre direction; ensuite, parce qu'il veut défendre la nature mortelle de l'Héraclès grec en opposition avec la nature divine de l'Héraclès égyptien. On sait aujourd'hui que la question est plus complexe et beaucoup de chercheurs se sont exprimés en faveur de l'origine grecque du mythe d'Héraclès ${ }^{35}$.

Le deuxième argument d'Hérodote dit ceci : si les Égyptiens avaient emprunté aux Grecs quelques divinités, elles auraient été maritimes puisque les deux peuples avaient une communication maritime; ne connaissant pas Poseidon et les Dioscures, ils ne peuvent avoir emprunté des dieux grecs et, par conséquent, Héraclès non plus. Or, on ne peut pas comprendre pourquoi les Égyptiens devaient préférer seulement des dieux maritimes, au cas où ils les auraient empruntés aux Grecs. Ce raisonnement peut d'ailleurs servir également un point de vue opposé : puisque les Égyptiens ne connaissaient pas suffisamment de divinités grecques parmi lesquelles des dieux si importants que sont Poseidon, Héra ou Hestia, pour le panthéon héllénique (comme il le dit en II, 50,3), on peut en déduire soit qu'ils les ont découverts eux-mêmes,

32 L.R. FARNELL, Greek Hero Cults and Ideas of Immortality, Oxford, 1970 [1921], p. 100. Cf. aussi M.P. NILSSON, The Mycenaean Origin of Greek Mythology, New York, 1963 [1932], p. 189; HOW-WELLS, op. cit. (n. 22), p. 187; W. PÖTSCHER, Herakles, in Kletne Pauly, II (1967), col. 1049; F. PRINZ, Herakles, in RE, Suppl. XIV (1974), col, 159; A.B. LLOYD, op. ctt. (n. 8), p. 203; BURKERT, Greek Religion, op. cit. (n. 16), p. 210; W. PÖTSCHER, Hera, Eine Strukturanalyse im Vergleich mit Athena, Darmstadt, 1987, p. 28 et n. 53.

33 LEGRAND, op.cit. (n. 31), p. 96: «Alkaios, père d'Amphitryon, et Elektryon, père d'Alcmène, étaient fils de Persée, descendant d'Aigyptos par Lynceus et de Danaos, frère d'Aigyptos, par Hypermnestre ".

34 C'est la version qui est avancée par la plupart des sources: HOM., Il., V, 631; XIV, 266; XVIII, 117-118; XIX, 132 et ailleurs, HÉS., Tb., 943; [HÊs.], Boucller, 27; PHÉRÉCYDE, FGrH, 3 F 13a, 13b Jacoby; HDT., FGrH 31 F 16 Jacoby; APOLLODORE, II, 4, 3; D.S. IV, 9, 1; PLAUTE, Amph., 112-119 et 760 $\mathrm{sq}$.

35 Cf, par exemple, FARNELL, op. cit. (n. 32), p. 104; NILSSON, Mycenaean Origin, op. cit. (n. 32), p. 206 sq., et Cults, Mytbes, Oracles, and Politics in Ancient Greece, Lund, 1951, p. 69; G.K., GALINSKY, The Herakles Theme, Oxford, 1972, p. 3; PRINZ, art. cit. (n. 32), col. 146 sq. et surtout 157-162; BURKERT, Greek Religion, op. cit. (n. 16), p. 208 sq.; P. LÉVÊQUE - A. VERBANCK-PIÉRARD, Héraclès, béros ou dieu?, in C. BONNET - C. JOURDAIN-ANNEQUIN (éds), Héraclès d'une rive à l'autre de la Méditerranée. Bllan et Perspectives. Actes de la Table Ronde de Rome (Academia Belgica / École française de Rome 15-16/9/1989), Bruxelles-Rome, 1992 (Études de Philologie, d'Archéologle et d'Histotre anciennes publiées par l'Institut historique belge de Rome, 28) p. 44 sq. 
soit qu'ils ont emprunté certains d'entre eux, comme Poseidon par exemple, en Libye (II, 50, 3). Pourquoi n'auraient-ils pas emprunté aussi Héraclès ailleurs ?

Sur ce point s'achève la première étape de son argumentation qui se

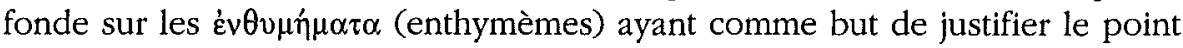
de vue selon lequel le mythe de l'Héraclès grec a été créé sous l'influence égyptienne. La deuxième étape, sa recherche de $\tau є \kappa \mu n ́ p ı$ (preuves certaines), s'ouvre avec la reformulation de la thèse qu'il posa dès le début de sa digres-

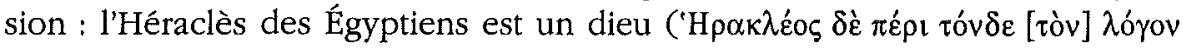

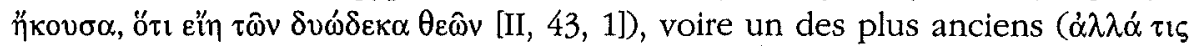

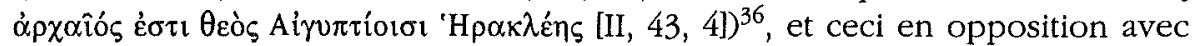
l'Héraclès grec qui est mortel et récent. Il a aussi l'occasion d'évoquer des témoignages archéologiques afin d'appuyer ses arguments, et prétend qu'il voyagea dans ce but à Tyr en Phénice où il vit, en plus du temple principal consacré à Hêraclès, un autre consacré à Héraclès Thasios. Selon lui, il existait à Thasos également un temple qui fut fondé par des Phéniciens, cinq générations avant que ne naisse en Grèce Héraclès, le fils d'Amphitryon. Par là, l'historien reconnaît aux Phéniciens un rôle d'intermédiaires pour l'introduction, en Grèce, du culte de l'Héraclès égyptien, ce qui le conduit à des estimations erronées. Il identifie ainsi Héraclès, l'ancien dieu des Égyptiens, comme il $\mathrm{dit}^{37}$, avec une figure phénicienne analogue, Melqart, qu'on adorait à $\mathrm{Tyr}^{38}$. Or, concernant le sanctuaire d'Héraclès à Tyr, on peut difficilement penser à une influence égyptienne; en revanche, il est très probable qu'il s'agit d'une implantation de son culte, de Tyr à Thasos, comme résultat des relations commerciales entre Thasos, la Phénice et l'Égypte ${ }^{39}$. Par ailleurs, la tradition selon laquelle les Phéniciens fondèrent un temple d'Héraclès à Thasos reflète leur extension maritime et leurs relations avec la Grèce qu'Hérodote envisage d'habitude comme la cause de nouvelles conceptions, de nouvelles mours et coutumes provenant d'Égypte et cela non sans raison ${ }^{40}$. Cependant, dans ce cas précis, il ne semble pas avoir raison: les chercheurs considèrent comme improbable

36 En ce qui concerne le problème religieux qui se pose ici concernant l'identité exacte d'Héraclès (qui est-il, quelle est sa place au sein du panthéon égyptien, quand et comment celui-ci fut crëé ?), voir HOW-WELls, op. cit. (n. 22), p. 187, 238-239; A.B. LLOYD, Commentary 1-98, op. cit. (n. 8), p. 201-202. Cf. EVANS, op. cit. (n. 27), p. 42.

37 En realité, il s'agit de Chonsu, selon A.B. LLOYD, ibid., p. 194-195, 202.

38 HOW-WELLS, op. cit. (n. 22), p. 188; FARNELL, op. cit. (n. 32), p. 142; A.B. LlOYD, op. cit. (n. 8), p. 205; EVANs, op. cit. (n. 27), p. 42; C. BONNET, Melqart. Cultes et mythes de l'Héraclès tyrien en Méditerranée, Namur-Leuven, 1988 (Blbliotbèque de la Faculté de Pbllosopbie et Lettres de Namur, 69. Studia Pboenicia, 8).

39 A.B. LlOYD, op. cit. (n. 8), p. 207-208, avec la biblographie sur le thème; BONNET, op. cit. (n. 38), p. 49-50, 351-352.

40 A.B. LloYD, Introduction, $o p$. ctt. (n. 1), p. 10, soutient que dès la fin de la période géométrique le commerce était aux mains des Phéniciens, alors «the Egyptian and Egyptianizing elements probably came to Greece through them ». Voir aussi FROIDEFOND, op. cit. (n. 23), p. 157. 
l'extension des Phéniciens avant le Xe siècle av. J.-C. ${ }^{41}$, alors qu'en se fondant sur les calculs d'Hérodote (cinq générations avant la naissance de l'Héraclès grec qu'il situe 900 ans avant son époque [II, 145, 4]), le temple devrait avoir été construit autour de $1500 \mathrm{av}$. J.-C. Ces données historiques en rapport avec les indices archéologiques relatifs au culte d'Héraclès ont conduit A. Lloyd à conclure que "clearly there is no case for a Phoenician origin of the Thasian Herakles ${ }^{42}$. Désormais sûr de lui quant à la nature divine et l'antériorité

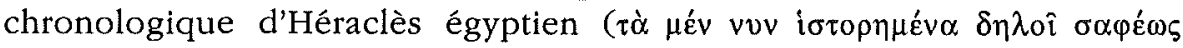

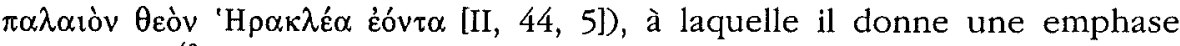
particulière ${ }^{43}$, Hérodote s'attaque violemment à la version grecque du mythe afin de la présenter comme indigne de foi. C'est une autre façon de renforcer sa position en tirant des conclusions a contrario, comme le faisaient habituellement les sophistes et surtout Gorgias : l'Héraclès égyptien est supérieur pour d'autres raisons encore que celles précédemment expliquées, mais également parce que l'Héraclès grec n'a pas d'attributs analogues. Il choisit la version qui se rapporte à la visite d'Héraclès en Égypte, où Bousiris essaya de le sacrifier, comme il le faisait avec tous les étrangers (II, 45). Dès que commença la cérémonie, Héraclès tua tous ceux qui étaient présents. La critique d'Hérodote se porte sur la naîveté du mythe et, par extension, sur le fait qu'il est indigne de foi ${ }^{44}$. Il essaye de démontrer sa thèse de deux façons : d'une part, en décelant dans le mythe les éléments qui ne s'accordent pas avec les coutumes égyptiennes (

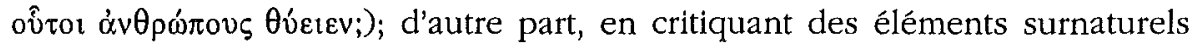

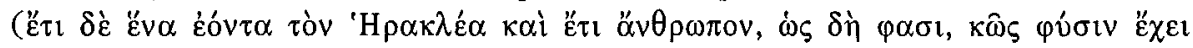

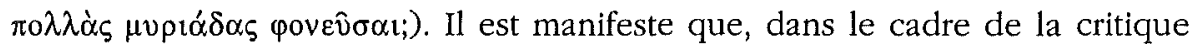
rationnelle des mythes datant de l'époque des logographes ioniens, mais qui est particulièrement répandue à l'époque d'Hérodote, le mythe en question est

41 On peut voir, par exemple, BURY-MEIGGs, op. cit. (n. 1), p. 64; U. WILCKEN, A $\rho \chi \alpha i \alpha$

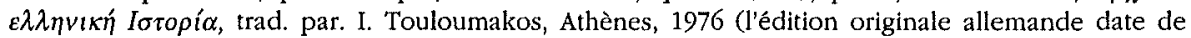
$1962^{9}$ ), p. 102; A.B. LLOYD, Commentary 1-98, op. cit. (n. 8), p. 209. On trouvera une opinion semblable chez DIETRICH, OMgins, op. cit. (n. 1), p. 73 et n. 14, où il discute rapidement ce thème, avec la bibliographie, et qui, en fait, ne favorise pas l'hypothèse de la présence des Phéniciens à l'Ouest durant le $2 \mathrm{e}$ millénaire av. J.-C.

42 Commentary, 1-98, op. ctt. (n. 8), p. 209. Cf, aussi p. 211. Selon BONNET, au contraire, « une implantation de Melqart à Thasos demeure possible, suivie d'une assimilation ... parfaite », p. 371. Mais cette implantation date du "premier quart du ler millénaire » av. J.-C., c'est-à-dire d'une époque évidemment plus tardive que celle calculée par Hérodote. Ainsi peut-on supposer qu'Hérodote transfert dans le passé les données d'une époque postérieure.



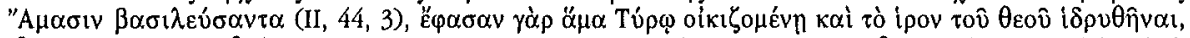

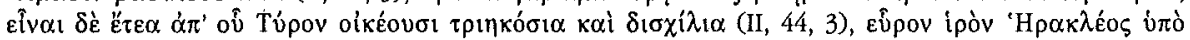

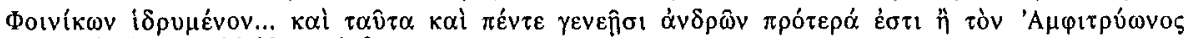

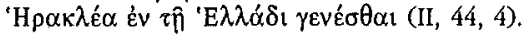

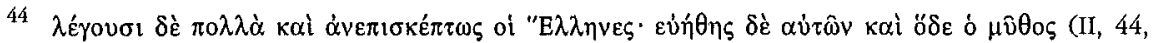
4) : l'historien utilise ici le mot $\mu \hat{v} \theta$ o૬ dans un sens négatif : «fable », « conte »: LSJ, p. 1151 (s.v. $\mu \hat{v} \theta 0 \varsigma) ;$ POWELL, op. cit. (n. 29), p. $228(s . v . \mu \hat{v} \theta$ o $)$. 
contesté du fait que ses éléments ne correspondent pas à la réalité, la religion égyptienne ne permettant pas de sacrifices humains. Cependant, comme le remarque J. Anastassiou, il est manifeste que les sacrifices humains du mythe de Bousiris ne constituent pas des souvenirs historiques d'un lieu concret, mais des actes liturgiques en vue d'apaiser le dieu et d'obtenir la pluie ${ }^{45}$. Et, dans tous les cas, le mythe peut difficilement être considéré comme une source historique. D'ailleurs, Hérodute passe sous silence le fait que la tradition grecque considère Héraclès, non pas comme un homme mais comme un héros et que, à l'instar de tous les héros, on lui attribue des exploits surnaturels.

En résumé, nous pouvons dire que le mythe d'Héraclès constitue un exemple représentatif de l'effort accompli par Hérodote pour imposer, sans base crédible et d'éléments suffisants, son point de vue sur la religion grecque. Il apparaît qu'il est lui-même conscient des difficultés; c'est pourquoi il porte un accent particulier tant sur la recherche d'arguments que sur l'organisation de l'argumentation. En ce qui concerne la recherche d'arguments, il choisit ceux qu'il considère comme étant les plus efficaces. Il utilise ainsi des arguments fondés sur la tradition (II, 43, 2) selon l'zíxó (II, 43, 3), les témoignages archéologiques (II, 44), ainsi que des arguments qui proviennent de son expérience (II, 45) ${ }^{46}$. Quant à l'organisation de l'argumentation, on peut y relever deux axes : l'un concerne les éléments qui séparent les deux figures différentes (opposition), celle de dieu, d'une part, et celle de l'homme mortel, d'autre part, - c'est une opposition qui revient constamment : en II, 43, 1-2 et plus loin en II, 43, 4 - II, 45 et en II, 44, 4-5); l'autre se rapportent à des éléments qui les unissent (analogie), comme l'origine commune et, à un certain degré, le culte. Dans ce contexte général, l'argumentation s'organise en fonction de l'espèce et du poids de ses arguments, selon trois unités : a) l'Héraclès égyptien n'a aucun rapport avec l'Héraclès grec, si ce n'est par le nom et par l'origine $(\dot{\varepsilon} \vee \theta v \mu \eta \dot{\mu} \mu \alpha \tau)$; b) l'Héraclès égyptien (opposé à l'Héraclès grec) est un dieu et même un dieu antique; son culte aurait été introduit en Grèce par les

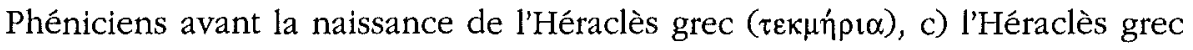
n'est qu'un mortel (conclusions a contrario). Bref, la volonté de l'historien de projeter à travers la confrontation continue, la version égyptienne d'Héraclès est bien manifeste.

Une fois qu'on discerne son argumentation, le problème se pose de savoir de quelle façon Hérodote relie les deux figures en question qui sont différentes alors même que l'historien rejette la conception grecque courante concernant Héraclès. En fin de compte qui est selon lui l'Héraclès grec : le dieu, l'homme, ou le héros? Bien plus, comment rencontre-t-il l'Héraclès égyptien? Pour avoir

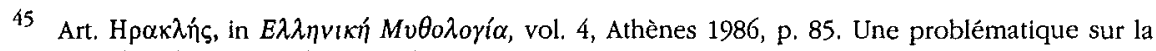
nature véritable de ces sacrifices est développée aussi par FROIDEFOND, op. cit. (n. 23), p. 178.

46 En ce qui concerne les modes d'argumentation d'Hérodote en général, voir A.B. LLOYD, Introduction, op. clt. (n. 1), p. 156-170. 
une réponse à ces questions nous devons tenir compte également des chapitres II, 145-146.

L'historien revient en effet sur le thème d'Héraclès dans le cadre de sa recherche sur l'histoire de l'Égypte et de ses rois. Héraclès, un des rois divins de l'Égypte, serait un ancien dieu égyptien, mais serait aussi, parmi les dieux grecs, un des plus récents, dont le culte fut introduit en Grèce par les Phéniciens $\pi \varepsilon ́ v \tau \varepsilon$

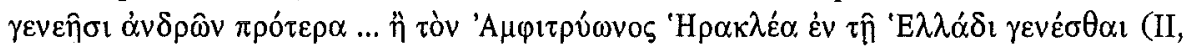
$44,4)^{47}$. En mémoire de cet événement, certains ${ }^{48}$ ont donné au fils d'Alcmène et d'Amphitryon, d'origine égyptienne, le nom du dieu, pour qu'il symbolise manifestement la provenance égyptienne de l'Héraclès-dieu. En conséquence, selon Hérodote, il existe en Grèce l'Héraclès - dieu et l'Héraclès - homme ${ }^{49}$, mais non l'Héraclès-héros, comme le veut la tradition grecque. ${ }^{50}$

47 Il situe la naissance d'Héraclès 900 ans avant son époque (II, 145, 4).

48 Il s'agit des poètes Homère et Hésiode, selon HOW-WWELLS, op. cit. (n. 22), p. 187.

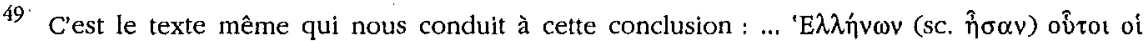

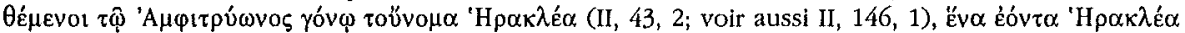

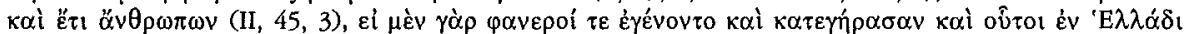

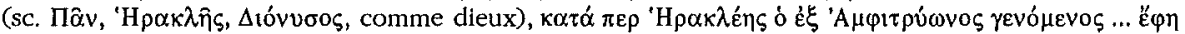

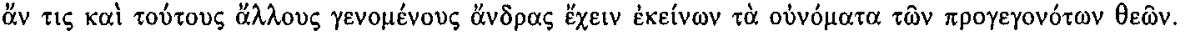
Pour l'emploi du mot ávíp ici en opposition avec le mot $\theta \varepsilon$ ćs, voir POWELL, op. cit. (n. 29), p. 26 $(s, \nu, \dot{\alpha} v \dot{\eta} \rho)$. Par ailleurs, étant donné la thèse d'Hérodote selon laquelle la plupart des phénomènes de la religion grecque sont d'origine égyptienne, l'historien ne pourrait pas accepter l'idée de l'Héraclès-héros, puisque, dans la religion égyptienne, il n'y a pas, d'après lui, de héros (II, 50, 3). Sur le même thème, voir FARNell, op. ctt. (n. 32), p. 98; J. Gould, Herodotus, London, 1989, p. 11.

50 Nous savons qu'Héraclès est un, sinon le plus important, des héros de la mythologie antique grecque, à qui on a attribué un nombre exceptionnel d'exploits: cf. ANASTASSIOU, op. cit. (n. \$), p. 121-122, qui écrit que beaucoup de héros de la mythologie grecque ont réalisé des exploits, mais tous ceux-ci demeuraient dans les limites de la nature humaine et avaient à faire à des hommes; le mythe d'Héraclès se caractérise par le fait qu'une grande partie de ses exploits est en rapport avec des êtres fantastiques et surnaturels, ainsi qu'avec des événements qui se situent au-delà des possibilités humaines. Le même auteur reconnaît dans le cycle mythique d'Héraclès la thématique du caractère du héros-demi-dieu. - Héraclès était donc d'abord un héros qui, après sa mort, est devenu dieu et non l'inverse : Od., II, 601; HÉs., Th., 950; APOLLODORE, XXVII, 7; D.S. IV, 38-39; OVIDE, Mét., IXX, 239 sq. et ailleurs. Cf. PAUS., II, 10, 1 et ARRIEN, Anab., IV, 11, 7. Sur le même thème, voir aussi FARNELl, op. cit. (n. 32), p. 98 sq.; NilSSON, Myceanean Origin, op. cit. (n. 32), p. 204-206; F. STOESSL, Der Tod des Herakles, Zürich 1945, p. 11 sq.; NILSSON, Der Flammentod des Herakles auf dem Otte, in Opuscula Selecta, vol. I, Lund, 1951, p. 348-354; GALINSKY, op. cit. (n. 35), p. 5-6; PRINZ, art. cit. (n. 32), p. 196; A.B. LloYD, Commentary 1-98, op. cit. (n. 8), p. 203. En ce qui concerne les témoignages archéologiques qui se réfèrent à la divinisation du héros, on peut consulter l'article de K. SCHAUENBERG, Herakles unter Gottern, in Gymnasium, 70 (1963), p. 113-133, avec une bibliographie abondante. La divinisation d'Héraclès constituerait un des éléments les plus récents du mythe (cf. ANASTASsIOU, op. cit. [n. \$], p. 119). D'autres chercheurs considèrent aussi comme des ajouts plus récents les passages de l'épopée qui parlent de divinisa-

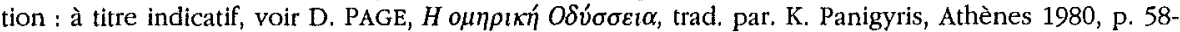
59; M.L. WEST (éd.), Hesiod Theogony with Prolegomena and Commentary, Oxford, 1966, p. 416417 pour les vers 947-955 de la Théogonte. Selon H.A. SHAPIRO, Hêrôs Theos: The Death and Apotbeosis of Herakles, in CW, 77(1983), p. 15 sq., le mythe de la divinisation a été inventé par les Grecs à une époque postérieure afin de justifier son culte comme dieu : «In areas which had at an early date subject to the influence of Near Eastern cults, Greeks would have found themselves worshipping Herakles in a dual cult, as god and hero, and would have felt them to create an explanation of its origins in purely Greek terms ». 
Il apparaît en fait qu'Hérodote tire profit du double culte d'Héraclès en tant que dieu et héros ${ }^{51}$. et surtout de la conception de la divinisation, dans le but de mettre en évidence la nature divine du héros, qu'il interprète avec sa méthode favorite, en insistant sur un emprunt à l'Égypte sur base de l'argument habituel qui consiste à faire de la religion égyptienne une religion plus ancienne que celle des Grecs ${ }^{52}$. Pour qu'Hérodote arrive à cette thèse d'un Héraclèsdieu, dégradé par la suite en héros ${ }^{53}$, il lui a fallu rejeter toute la tradition grecque dans laquelle Héraclès suit un cheminement inverse; c'était un héros qui finalement a été divinisés4. Un autre cas de forme mixte peut être décelé dans la fête en l'honneur de Dionysos que les Égyptiens organisent pratiquement de la même façon que les Grecs (analogie), exception faite des danses et, à un certain degré, des phallus (opposition) ${ }^{55}$.

Pour Hérodote, ces ressemblances et différences qui pourraient être dues au hasard ou à une évolution parallèle d'un phénomène religieux analogue ou encore à une influence grecque, conduisent de nouveau à l'Égypte (II, 49, 1-2). Néanmoins, par la démarche qu'il suit, sa thèse apparaît clairement son effort de couvrir le manque d'éléments démonstratifs en développant un syllogisme habile caractérisé par une stratégie concrète : mettre en évidence le rôle de Melampous, sur lequel il fonde sa théorie de l'introduction du culte du dieu en Grèce. Il le fait d'une manière si méthodique que ce rôle devient auto-démontrable, le but final étant de rendre caduques toutes les positions adverses. ${ }^{56}$

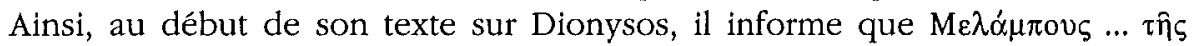

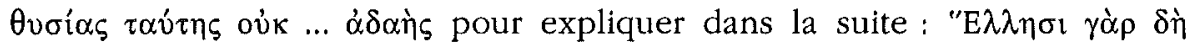

51 FARNELl, op. cit. (n. 32), p. 96 sq.; WEST, op, cit. (n. 50), p. 416-417; A.B. LlOYD, op. cit. (n. 8), p, 208-209 et 211-212. Selon Farnell et West, le culte d'Héraclès-héros était plus ancien que le culte d'Héraclès-dieu, qu'on fait remonter au VIe siècle avant J.-C. pour la région de l'Attique : voir par exemple FARNELL, op. cit. (n. 32), p. 108; WEST, op. clt. (n. 50), p. 417; A. VERBANCK-PIÉRARD, Le double culte d'Héraklès: légende ou réalité, in A.F. LAURENS (éd.), Entre bommes et dieux. Le convive, le béros, le prophète, Besançon-Paris, 1989, p. 64. À ce double culte fait écho la passage :

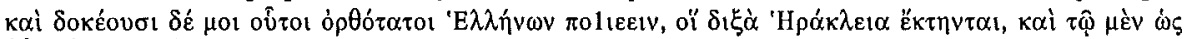

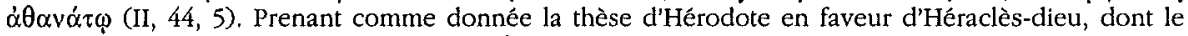
culte aurait été repris par les Grecs en Égypte par l'intermédiaire des Phéniciens à une époque ancienne, nous devons supposer que l'historien entend par là que ce dieu a été dégradé plus tard en héros; c'est pourquoi il était adoré comme tel.

52 Il s'agit d'un argument $a b$ antiquiore, qui parcourt tout le livre II (Voir aussi FROIDEFOND, op. clt. [n. 23], p. 147) ou, comme A.B. LLOYD le dit, le sophisme de post boc ergo propter boc (op. cit. [n. 8], p. 147).

53 Cf. FARNELL, op. cit. (n. 32), p. 97, bien que PÖTSCHER, art. cit. (n. 32), col. 1052 et SHAPIRO, art. cit. (n. 50), p. 11, acceptent la divinisation d'Héraclès et parlent d'une double nature du dieu. Celle-ci résulte, selon Shapiro, de quelques éléments de son mythe (par exemple sa descente dans l'Hadès pour ramener Cerbère, les bœufs de Géryon, ou encore les pommes des Hespérides).

54 Cf. supra, n. 50.

55 II, 48, 1-2.

56 Cf. FROIDEFOND, op. ctt. (n. 23), p. 153, qui a aussi perçu la partialité d'Hérodote, reflétée dans son argumentation : "La diversité des arguments invoqués dans des cas semblables par Hérodote pour défendre la thèse de l'origine égyptienne, dénote, malgré une rigueur logique apparente, un embarras qui peut être le signe d'une certaine partialité ». 


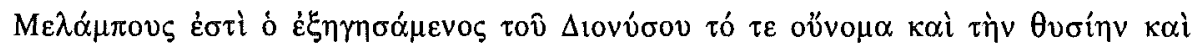

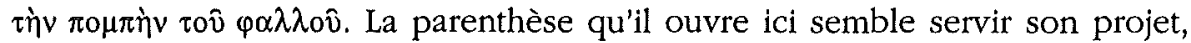

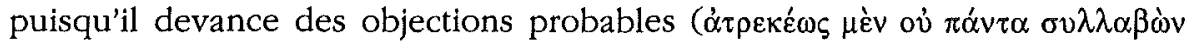

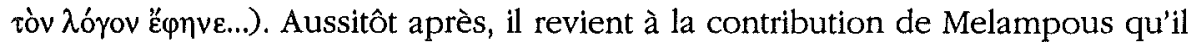
prend soin d'accentuer en créant avec ce qui précède une forme de discours

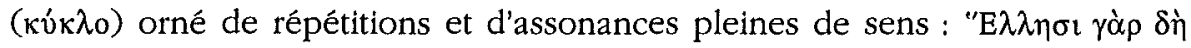

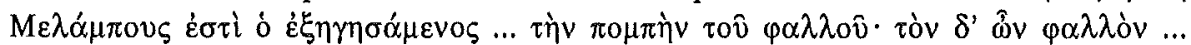

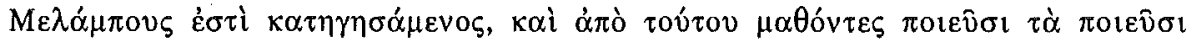

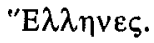

Sa thèse il la formule clairement à la fin, comme conséquence du

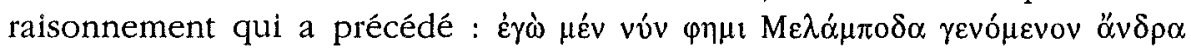

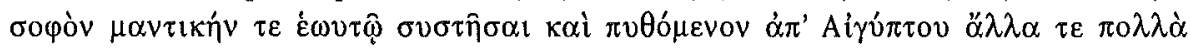

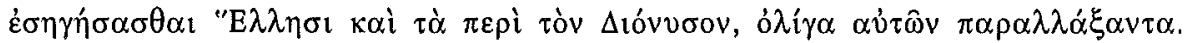
L'historien sait que sa conclusion est subjective, et en assume la responsabilité :

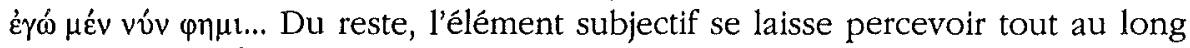

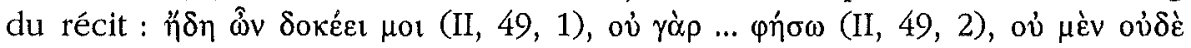

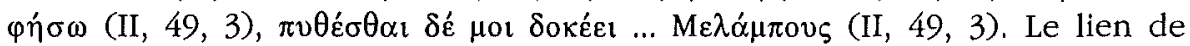
Melampous avec l'Égypte doit être une idée d'Hérodote ${ }^{57}$. Il est arrivé à cette conclusion en poursuivant un processus logique éclairé par le principe d'analogie : les Égyptiens avaient depuis longtemps un dieu Dionysos (II, 145) ${ }^{58}$ et organisaient une fête en son honneur avec diverses manifestations. Melampous aurait connu tout ce qui avait rapport avec Dionysos et qui est arrivé en Grèce à une époque ultérieure ( $v \varepsilon \omega \sigma \tau i ~ \varepsilon ́ \sigma \eta \gamma \mu \varepsilon ́ v \alpha$ ). Il est donc très probable qu'il ait été instruit sur ce sujet en Égypte, puisque c'est de là que sont parvenus également d'autres phénomènes religieux (par exemple le culte du dieu-Héraclès). Dans le cadre de ce raisonnement, la phrase ó $\lambda \hat{i} \gamma o v \pi \alpha \rho \alpha \lambda \lambda \alpha_{\alpha}^{\prime} \xi \alpha v \tau \alpha$ a pour objectif de justifier les divergences sur le thème, entre les deux peuples ${ }^{59}$, afin qu'elles ne puissent constituer un obstacle à sa théorie.

L'historien a peut-être pris conscience du fait que le processus de démonstration utilisé jusqu'à présent n'est pas suffisant; aussi cherche-t-il à renforcer sa position en rejetant les autres versions comme dénuées de fondement. Comme

57 Ce n'est pas la première fois que Melampous est relié à Dionysos [voir PLEY, Melampus, in RE, XV, 1 (1931), col. 398; BURKERT, Über die Namen der Götter, art. ctt. (n. 28), p. 122, n. 3], mais c'est la première fois, me semble-t-il, qu'il est mis en relation avec l'Égypte. Cf. M. JOST, in Polydipsion Argos. Argos de la fin des palats mycéntens à la constitution de l'État classique, Fribourg (Suisse) 7-9 mal 1987, p. 175. Le lien entre l'Égypte et Melampous est réalisé par un Phénicien, Cadmos le Tyrien.

58 Comme le remarque A.B. LLOYD, op.cit. (n. 8), p. 224, ici aussi on doit faire la distinction entre le dieu Dionysos des Égyptiens et le fils de Sémélé, comme ce fut le cas pour Héraclès. Le Dionysos en question est le dieu égyptien que Cadmos a apporté en Grèce avant la naissance du fils de Sémélé.

59 Hérodote avait déjà remarqué des différences concernant les danses et la procession du

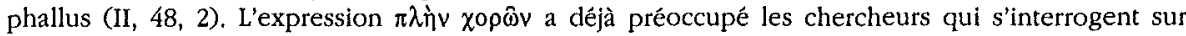
son sens exact, puisque dans chaque cas elle marque une différence essentielle dans le culte du dieu : A.B. LLOYD, ibid., p. 220. 
W. Burkert l'a déjà remarqué : « Herodot natürlich kein syllogistisches Schema benützt, sodern die 'eleatische' Form der Argumentation, die seit Parmenides und Zenon durch 'Fallunterscheidung' und reductio ad absurdum geprägt ist ${ }^{60}$. La possibilité de l'emprunt inverse, Hérodote l'a exclue sans aucune

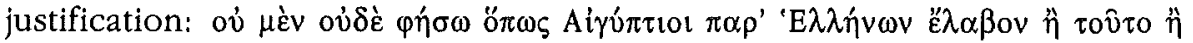
ă $\lambda \lambda_{0}$ кov́ $\tau$ vó $\mu \alpha$ tov (II, 49, 3); tandis que dans le cas de l'occurrence, il

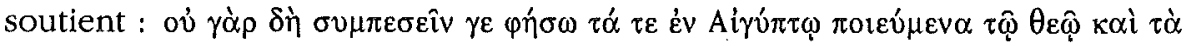

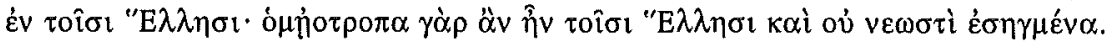

Cependant, on s'en doute, le bien-fondé de ses affirmations doit encore une fois être examiné. En ce qui concerne l'ó $\mu$ ó $\rho_{0} \pi \alpha^{61}$, l'historien semble avoir raison lorsqu'il soutient que les traditions concernant Dionysos et son culte contiennent des influences étrangères, mais ces influences ne sont pas nécessairement ou, du moins, exclusivement égyptiennes ${ }^{62}$. D'ailleurs, Hérodote luimême mentionne Dionysos comme un dieu parmi les plus importants des Thraces $(V, 7)$, contribuant ainsi à établir l'opinion d'une provenance thrace de

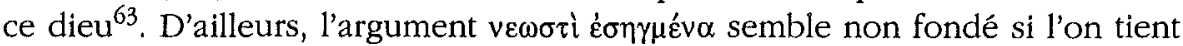
compte de nouvelles données scientifiques: le déchiffrage du nom de

\footnotetext{
60 BURKERT, Herodot aber die Namen der Götter, art, cit. (n. 28), p. 122.

61 " Ce culte devrait être en harmonie avec les mours des Grecs » (selon la traduction de Legrand, p. 101).

62 Selon DIETRICH, Origins, op. cit. (n. 1), p. 1-68, la création de l'ancienne religion grecque a été influencée par des croyances, modèles et pratiques orientales et particulièrement ceux des peuples du Nord-Ouest de l'Orient avec lesquels les Mycéniens (le peuple qui, selon son point de vue, a créé les aspects principaux de la religion grecque classique) avaient des relations étroites par l'intermédiaire de la Crète. C'est à ce cadre général qu'appartient aussi tout ce qui a rapport avec Dionysos et son culte : cf. p. 179 et ailleurs. BURKERT, Greek Religion, op. cit. (n. 16), p. 163, considère comme probable le fait que les plus anciennes relations cilico-syriaques ont été recouvertes (dans le mythe de Dionysos) par les traditions phrygiennes et, par la suite, lydiennes, tandis qu'il n'exclut pas des influences provenant de la religion égyptienne d'Osiris, mais qu'il les

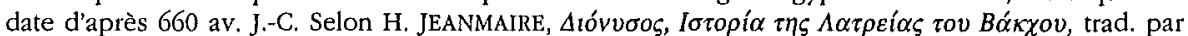
A. Mertani-Lisa, Athènes, 1985 (l'édition originale française date de 1951), p. 31-32, le dieu Dionysos est arrivé en Grèce, peut-être d'Asie Mineure. E. SIMON, Die Götter der Griechen, München, 1985 ${ }^{3}$, p. 289 , rejette la provenance lydo-phrygienne de Dionysos et soutient que les Grecs ont pris de la Phrygie seulement une forme parallèle tardive de Dionysos, le Sabazios, qui est introduit à Athènes à l'époque classique. Cependant, elle fait ici allusion à une influence hittite lorsque elle se rapporte, d'une part, à des îles grecques (Lesbos, Chios, Samos) ayant une longue tradition dans la production du vin - un produit qu'aimaient bien leurs voisins Hittites - et, d'autre part, au lien qui existe entre le vin et le dieu Dionysos. Parallèlement il parle aussi d'une provenance thrace du dieu. Cf. infra, note 64. Enfin, contrairement aux opinions qui favorisent une provenance thracophrygienne du dieu, W.F. OTTO, dans son livre Dlonysos, Mythos und Kultus, Frankfurt, $1960^{3}$ [1933], p. 51-62, et surtout p. 56, soutient qu'il s'agit d'un dieu grec indigène qu'on peut dater au moins de la fin du $2 \mathrm{e}$ millénaire av. J.-C.

63 C'est un point de vue que E. ROHDE, Psycbé, Paris, 1928, a fondé en se basant, entre autres, sur le témoignage d'Hérodote que nous avons déjà mentionné : il soutient que Dionysos était une divinité postérieure qui fut introduite en Grèce depuis la Thrace. Le même point de vue a été défendu par d'autres chercheurs également, comme par exemple NILSSON GGrR, vol. I, p. 564-568, qui cependant accepte aussi une origine phrygienne du dieu du côté de sa mère.
} 
Dionysos sur les tablettes du Linéaire $\mathrm{B}^{64}$ a posé un problème aux chercheurs,qui discutent à présent de l'éventualité d'un ancien dieu grec dont l'origine date de l'époque créto-mycénienne et plus haut encore ${ }^{65}$. L'éventualité d'influences dans le culte de Dionysos issues du culte égyptien d'Osiris à une époque ultérieure n'est pas négligeable ${ }^{66}$. Cependant, cela ne signifie pas qu'Hérodote a le droit de tirer de conclusions générales quant à une origine étrangère du dieu et de son culte.

Comme A. Lloyd l'indique, ayant désormais pris position en faveur de l'emprunt, Hérodote essaie de trouver le mode sous lequel l'emprunt s'est produit $^{67}$. Il le réussit en reliant Melampous avec l'Égypte à travers Cadmos. En

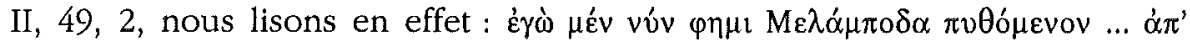



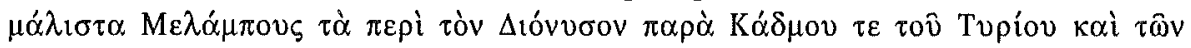

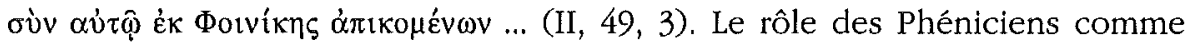
des intermédiaires est remis en évidence, mais, cette fois encore, il est difficile de considérer son information comme digne de foi. Comme il le dit lui-même,

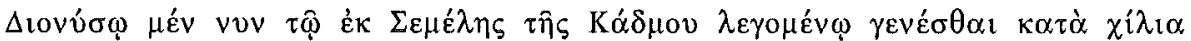

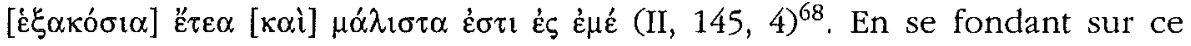
calcul, le culte du dieu a été introduit en Grèce au milieu du 2e millénaire av. J.-C. au plus tard. Mais comme nous l'avons déjà vu, la présence des Phéniciens en Occident à cette époque est sérieusement contesté ${ }^{69}$.

64 DIETRICH, Origins, op. cit. (n. 1), p. 175-176; C. KERÉNYI, Dionysos, Arcbetypal Image of Indestructible Life, trad. par R. Manheim, London, 1976, p. 68; SIMON, op. cit. (n. 62), p. 270; BURKERT, Greek Religion, op. cit. (n. 16), p. 45, 162.

65 KERÉNYI, op. cit. (n. 64), p. 5-125 et surtout 52 sq. MUTH, op. cit. (n. 28), p. 113 sq., parle d'un dieu mycénien qui était probablement aussi adoré en Crète. Le culte de ce dieu, pense-t-il, fait partie de la couche préhéllénique. Dans ce contexte, le rôle de Melampous comme fondateur du culte de Dionysos en Crète constitue, toujours selon le même auteur, un indice de plus qui favorise sa conclusion : l'existence d'un culte grec de Dionysos à l'époque mycénienne. D'autre part, selon SIMON, Dionysos était connu sous plusieurs figures dont deux étaient les plus importantes : l'une en fait un dieu des Ménades, d'origine thrace, que les Grecs auraient amené avec eux lorsqu'ils sont venus s'installer en Grèce; ils lui donnèrent pour patrie la Béotie et l'honoraient lors des Lénaia et des Dionysia; l'autre en fait un dieu préhéllénique de la végétation et tout particulièrement un dieu de la vigne, le seigneur des îles de la mer Égée ayant été lié avec la Crète, par l'intermédiaire de son épouse Ariane. Ce Dionysos était honoré à Anthestiria. Ces deux figures de Dionysos, dit-elle, bénéficiaient d'honneurs comparables et recouvraient, avant tout, le même dieu puissant. Les tablettes de la région de Chania, font d'ailleurs voir que Dionysos aurait été introduit dans le panthéon grec à l'époque de Bronze, comme fils de Zeus: voir E. HALLAGER - M. VLASAKIS - B.P. HALLAGER, New Linear B Tablets from Kbania, in Kadmos, 31 (1992), p. 76 sq.

66 Voir BURKERT, Greek Religion, op. cit. (n. 16), p. 163.

67 Vol. 2, p. 224

68 Il s'agit d'un cas analogue à celui d'Héraclès : l'historien distingue ici Dionysos-dieu que Cadmos a apporté en Grèce et Dionysos-mortel, le fils de Sémélé, fille de Cadmos, qui est né plus tard, au moins mille ans avant l'époque d'Hérodote, et qui a pris le nom du dieu, en mémoire de l'introduction de son culte en Grèce (II, 145-146).

69 Cf. supra, n. 41. 
Je pourrais extraire de l'œuvre d'Hérodote d'autres cas encore qui falisifient les influences culturelles et religieuses entre la Crète et l'Égypte. Mais je crois avoir traité des cas les plus importants. Avant de conclure, je me permets néanmoins de souligner que des structures logiques comme celles des couples opposés et des ressemblances logiques rendent plus claire l'argumentation de notre auteur. Hérodote s'emploie à fonder par ces moyens la supériorité culturelle et religieuse des Égyptiens. Certes, l'usage d'un argument inverse, c'est-à-dire celui qui soutient l'emprunt de la religion égyptienne à la religion grecque, est en principe rejeté par Hérodote, et il est même refusé catégoriquement : II, 49, 3 et II, 31, 1. Malgré tout cela il se voit obligé d'admettre une influence grecque pour les fêtes en l'honneur de Persée à Chemmis d'Égypte. Nous devons également présumer une influence grecque un peu plus bas (II, $92,1)$ où il rapporte que les Égyptiens des marécages ont généralement les mêmes coutumes que les autres, mais vivent avec une seule femme, comme les Grecs. Par ailleurs, même si nous négligeons les contacts entre les deux peuples, qui datent d'une époque ancienne, il est impossible qu'il n'y ait pas eu d'influences grecques sur la civilisation égyptienne, lorsque, selon le témoignage d'Hérodote lui-même, le roi Amasis, ami des Grecs, leur a cédé la ville de Naucratis et leur a donné des lieux pour qu'ils construisent autels et sanctuaires en l'honneur de leurs dieux (II, 178, 1).

Avec tout ce que j'ai retenu jusqu'à présent, je n'ai pas l'intention de nier toute influence égyptienne sur la civilisation grecque. Je veux seulement montrer que les influences n'ont pas forcément pris une seule direction, comme le veut Hérodote, qui, ébloui par le grandeur et l'ancienneté de la civilisation égyptienne, attribue à celle-ci toute réussite allant jusqu'à parler des Grecs d'une façon dépréciative. C'est, peut-être, pour cette raison que, durant l'époque alexandrine, la critique antique a été particulièrement sévère envers lui, en l'accusant d'être un «ami des barbares ${ }^{70}$.

G. ZOGRAPHOU

Université de Jannina

Faculté des Lettres

GR - 45110 JANNINA

70 A. MOMIGLIANO, The Place of Herodotus in the History of Historiography, in Studies in Historiography, London, 1966, p. 139. 